\title{
TU/e EmonONEN

\section{A robust soliton ratchet using combined antiferromagnetic and ferromagnetic interlayer couplings}

\section{Citation for published version (APA):}

Mansell, R., Lavrijsen, R., Fernández-Pacheco, A., Petit, D. C. M. C., Lee, J. H., Koopmans, B., Swagten, H. J. M., \& Cowburn, R. P. (2015). A robust soliton ratchet using combined antiferromagnetic and ferromagnetic interlayer couplings. Applied Physics Letters, 106, 092404-. https://doi.org/10.1063/1.4914014

DOI:

10.1063/1.4914014

Document status and date:

Published: 01/01/2015

\section{Document Version:}

Publisher's PDF, also known as Version of Record (includes final page, issue and volume numbers)

\section{Please check the document version of this publication:}

- A submitted manuscript is the version of the article upon submission and before peer-review. There can be important differences between the submitted version and the official published version of record. People interested in the research are advised to contact the author for the final version of the publication, or visit the $\mathrm{DOI}$ to the publisher's website.

- The final author version and the galley proof are versions of the publication after peer review.

- The final published version features the final layout of the paper including the volume, issue and page numbers.

Link to publication

\section{General rights}

Copyright and moral rights for the publications made accessible in the public portal are retained by the authors and/or other copyright owners and it is a condition of accessing publications that users recognise and abide by the legal requirements associated with these rights.

- Users may download and print one copy of any publication from the public portal for the purpose of private study or research.

- You may not further distribute the material or use it for any profit-making activity or commercial gain

- You may freely distribute the URL identifying the publication in the public portal.

If the publication is distributed under the terms of Article 25fa of the Dutch Copyright Act, indicated by the "Taverne" license above, please follow below link for the End User Agreement:

www.tue.nl/taverne

Take down policy

If you believe that this document breaches copyright please contact us at:

openaccess@tue.nl

providing details and we will investigate your claim. 


\section{AIP Apoled Phosics \\ Letters}

\section{A robust soliton ratchet using combined antiferromagnetic and ferromagnetic interlayer couplings}

R. Mansell, R. Lavrijsen, A. Fernández-Pacheco, D. C. M. C. Petit, J. H. Lee, B. Koopmans, H. J. M. Swagten, and R. P. Cowburn

Citation: Applied Physics Letters 106, 092404 (2015); doi: 10.1063/1.4914014

View online: http://dx.doi.org/10.1063/1.4914014

View Table of Contents: http://scitation.aip.org/content/aip/journal/apl/106/9?ver=pdfcov

Published by the AIP Publishing

\section{Articles you may be interested in}

Interfacial effects on magnetic interlayer coupling between perpendicular Co/Ni multilayers across Ru spacer Appl. Phys. Lett. 105, 262403 (2014); 10.1063/1.4905244

Readout method from antiferromagnetically coupled perpendicular magnetic recording media using ferromagnetic resonance

J. Appl. Phys. 114, 213901 (2013); 10.1063/1.4832827

Balancing interlayer dipolar interactions in multilevel patterned media with out-of-plane magnetic anisotropy Appl. Phys. Lett. 94, 052503 (2009); 10.1063/1.3078523

Perpendicular interlayer coupling through oscillatory Ruderman-Kittel-Kasuya-Yosida interaction between Co/Pt multilayers and $\mathrm{Co} / \mathrm{Tb}$ Co bilayers

J. Appl. Phys. 101, 09D121 (2007); 10.1063/1.2712958

Interlayer coupling and magnetic reversal of antiferromagnetically coupled media

Appl. Phys. Lett. 80, 91 (2002); 10.1063/1.1431397

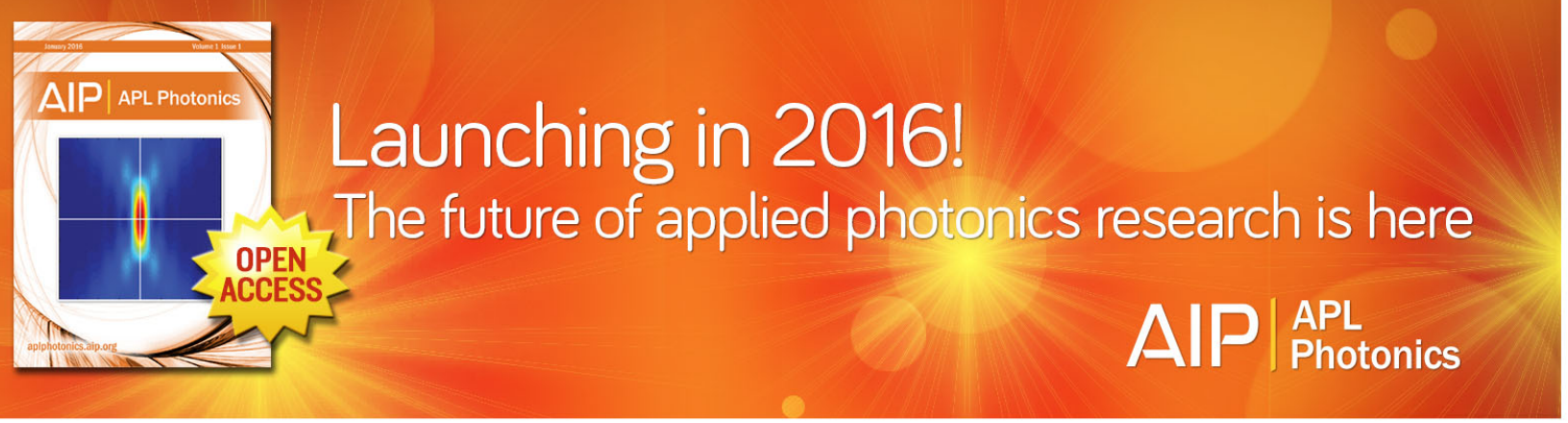




\title{
A robust soliton ratchet using combined antiferromagnetic and ferromagnetic interlayer couplings
}

\author{
R. Mansell, ${ }^{1}$ R. Lavrijsen, ${ }^{2}$ A. Fernández-Pacheco, ${ }^{1}$ D. C. M. C. Petit, ${ }^{1}$ J. H. Lee,${ }^{1}$ \\ B. Koopmans, ${ }^{2}$ H. J. M. Swagten, ${ }^{2}$ and R. P. Cowburn ${ }^{1}$ \\ ${ }^{1}$ Cavendish Laboratory, University of Cambridge, JJ Thomson Avenue, Cambridge CB3 OHE, United Kingdom \\ ${ }^{2}$ Department of Applied Physics, Eindhoven University of Technology, Den Dolech 2, 5612 AZ, P.O. Box 513, \\ Eindhoven, The Netherlands
}

(Received 19 December 2014; accepted 22 February 2015; published online 3 March 2015)

\begin{abstract}
A sharp magnetic soliton can be created and propagated in a vertical ratchet structure based on magnetic layers with out-of-plane anisotropy using a combination of antiferromagnetic and ferromagnetic interlayer couplings. This allows the use of identical magnetic layers in the stack, which simplifies the implementation of the ratchet compared to schemes which use alternating layer thicknesses. The ratchet behavior is analyzed using an Ising-macrospin approximation and conditions are derived for the propagation of a soliton, which is demonstrated experimentally. Values extracted from the experimental data for the coercivities and interlayer couplings show significant variation, which demonstrates the robustness of the soliton propagation. (C) 2015 AIP Publishing LLC.

[http://dx.doi.org/10.1063/1.4914014]
\end{abstract}

Three-dimensional spintronic devices, with the ability to propagate information out of the plane, would allow the creation of very high density storage media and novel forms of logic. ${ }^{1-3}$ One way to create a data bit in such devices is through an elementary excitation from the ordered ground state. This can be considered to be a domain wall, separating regions with opposite magnetism in the simplest case, or dividing the two energetically degenerated states in antiferromagnetically coupled lattices. ${ }^{2-8}$ This form of domain wall, which can occur in both continuous and discontinuous systems, is a magnetic kink soliton, which is referred to as a soliton in this work. ${ }^{1,6,9}$ Two main classes of magnetic materials can be used, shape anisotropy dominated and perpendicularly magnetized. For shape dominated materials, a chirality of rotation can be defined in the wall separating the two states. ${ }^{10,11}$ Using Ruderman-Kittel-Kasuya-Yosida (RKKY) interlayer coupling or dipolar interactions, it is possible to create vertical data storage schemes using in-plane materials with two-dimensional degrees of freedom that exploit this chirality. ${ }^{8,10,12}$ A different type of system can be made using perpendicular materials where, in the limit of large anisotropy and sharp domain wall mediated coercive switching, the system becomes Ising-like ${ }^{13}$ and is effectively restricted to one degree of freedom. However, reducing the system to one degree of freedom means that using chirality to break the symmetry is not possible. This leads to the idea of creating a ratchet which allows only unidirectional propagation. ${ }^{1,14,15}$ We have previously demonstrated a vertical ratchet scheme which uses entirely antiferromagnetic coupling between perpendicularly magnetized layers, using alternating layer thickness and interlayer coupling strengths. ${ }^{1,16}$ In this paper, we demonstrate that a repeated sequence of antiferromagneticantiferromagnetic-ferromagnetic (AF-AF-F) coupled magnetic layers creates a synchronous vertical soliton ratchet whilst using layers of the same material and thickness. We show theoretically how this scheme can support solitons across both antiferromagnetically and ferromagnetically coupled layers creating a generalized ratchet scheme. The ferromagnetic coupling breaks the symmetry of the device giving unidirectionality, which three (or more) AF couplings with magnetic layers of the same material and thickness cannot do, even if the coercivity is allowed to vary freely (see supplementary material), ${ }^{17}$ meaning that no repeating motif soliton ratchet device is possible. Having identical magnetic layers is an advantage of this scheme, since different thickness magnetic layers have different coercivity responses in terms of frequency, ${ }^{18-20}$ so although the coercivities can be similar on typical laboratory timescales of, for example, magneto-optical Kerr effect (MOKE) measurements, they may be different on fast, technologically relevant timescales. For these ratchet schemes, large variations in coercivity between layers can lead to the breakdown of ratchet behavior. Also, in the scheme demonstrated here, all of the designed variation needed to create the ratchet is in the interlayer coupling which makes the optimization of the magnetic layer simpler. Experimentally, we use an eight magnetic layer stack to demonstrate soliton propagation, creating a soliton ratchet where combined switching of the ferromagnetically coupled layers leads to equivalent behavior to that demonstrated using varying thickness layers.

We show the two degenerate ground states of the AFAF-F coupling scheme in the two left-hand columns of Figure 1. The ground states are formed by configurations which contain no unsatisfied couplings, with a soliton created at the conjunction of these two states. The system is symmetric under the inversion of all the layers, leading to the three distinct solitons shown in the columns labelled (i), (ii), and (iii) in Figure 1. We analyze the transitions for the two layers which form the soliton across the three possible interlayer couplings, labelled $J_{A F 1}, J_{A F 2}$, and $J_{F M}$. We assume that each layer undergoes a complete easy axis switch at one field, an Ising-macrospin approximation, and those layers are coupled by RKKY interactions, which allow controlled ferromagnetic and antiferromagnetic coupling 


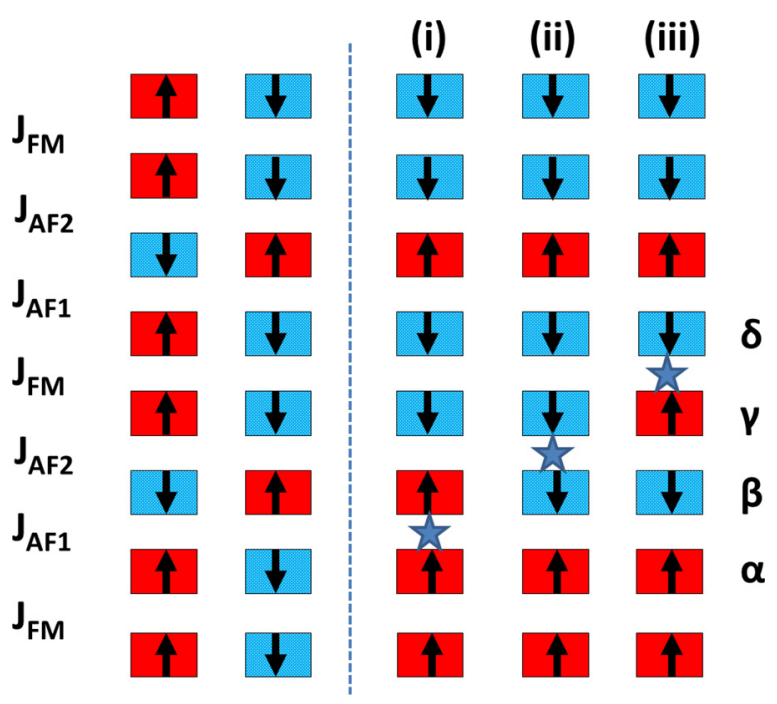

FIG. 1. A schematic of an eight layer AF-AF-F ratchet stack. The two left hand columns show the two degenerate ground states of the system. The interlayer couplings are labelled on the left hand side. On the right hand side, we show the three soliton positions possible in the sample. Column (i) shows the soliton across a $J_{A F 1}$ coupling formed of two layers labelled $\alpha$ and $\beta$. Column (ii) shows the soliton after correct propagation from column (i), now across $J_{A F 2}$ formed by layers labelled $\beta$ and $\gamma$. Column (iii) shows the soliton across the $J_{F M}$ coupling formed by layers $\gamma$ and $\delta$.

between nearest neighbor magnetic layers. In order to simplify the equations we define a quantity $J^{*}=J / M_{s} t$, which is the effective field from the interlayer coupling, where $J$ is the interlayer coupling surface energy density, $M_{s}$ is the magnetization saturation, and $t$ is the layer thickness. First, in Figure 1, column (i), we have a soliton across the $J_{A F 1}$ coupling layer between the magnetic layers labelled $\alpha$ and $\beta$. For simplicity, we use the modulus of the coupling and use the preceding sign to give the correct switching field. For layer $\alpha$, we can write the switching field as

$$
H_{\alpha}=-H^{c}+\left|J_{A F 1}^{*}\right|-\left|J_{F M}^{*}\right|
$$

with $H^{c}$ the coercivity of the layer, which we take to be the same for all layers. Similarly, for layer $\beta$ we have

$$
H_{\beta}=-H^{c}+\left|J_{A F 1}^{*}\right|-\left|J_{A F 2}^{*}\right| .
$$

Note that $H^{c}$ is negative in these equations because we are switching from up to down. This means that layer $\beta$ will switch first as long as $\left|J_{F M}^{*}\right|>\left|J_{A F 2}^{*}\right|$. Similarly, for column (ii)

$$
\begin{gathered}
H_{\beta}=H^{c}+\left|J_{A F 1}^{*}\right|-\left|J_{A F 2}^{*}\right|, \\
H_{\gamma}=H^{c}+\left|J_{F M}^{*}\right|-\left|J_{A F 2}^{*}\right|,
\end{gathered}
$$

where the equation for $H_{\beta}$ has changed because that layer has switched compared to the previous step. This leads to $H_{\gamma}$ switching first if $\left|J_{F M}^{*}\right|<\left|J_{A F 1}^{*}\right|$, so that we require $\left|J_{A F 2}^{*}\right|<\left|J_{F M}^{*}\right|<\left|J_{A F 1}^{*}\right|$. The case of the soliton across the $\mathrm{F}$ coupling (column (iii)) requires us to distinguish between two cases. Layer $\delta$ can switch at higher fields than $\gamma$, so from the previous step the field is simply increased further to complete a cycle of the ratchet. This gives a three step ratchet with a soliton across the F coupling for some range of field. Second, it is possible for layer $\delta$ to switch at the same time as $\gamma$. This gives two step propagation and is equivalent to the ratchet using varying thickness layer previously demonstrated. ${ }^{1}$ The switching fields assuming a soliton across $J_{F M}$ are

$$
\begin{gathered}
H_{\gamma}=-H^{c}+\left|J_{F M}^{*}\right|-\left|J_{A F 2}^{*}\right|, \\
H_{\delta}=H^{c}+\left|J_{A F 1}^{*}\right|-\left|J_{F M}^{*}\right| .
\end{gathered}
$$

Whether $J_{F M}$ is closer in magnitude to $J_{A F 1}$, in which case layer $\gamma$ will switch with layer $\delta$, or closer to $J_{A F 2}$, in which case the soliton is stable across the ferromagnetically coupled layer, determines the behavior of the ratchet. Equations (5) and (6) also show that there is a link between coercivity and coupling strength in this ratchet scheme. Further, the switch of the most antiferromagnetically coupled layer, $\beta$, when the soliton is across $J_{A F 1}$, must occur at more negative field than any other of the propagation switches because otherwise the soliton will be able to escape the whole stack. This leads to the conditions

$$
\begin{aligned}
H_{\beta} & =-H^{c}+\left|J_{A F 1}^{*}\right|-\left|J_{A F 2}^{*}\right| \\
<H_{\gamma} & =H^{c}+\left|J_{F M}^{*}\right|-\left|J_{A F 2}^{*}\right|, \\
H_{\beta} & =-H^{c}+\left|J_{A F 1}^{*}\right|-\left|J_{A F 2}^{*}\right| \\
<H_{\delta} & =H^{c}+\left|J_{A F 1}^{*}\right|-\left|J_{F M}^{*}\right|,
\end{aligned}
$$

which rearranges to

$$
\begin{aligned}
& \left|J_{A F 1}^{*}\right|-\left|J_{F M}^{*}\right|<2 H^{c}, \\
& \left|J_{F M}^{*}\right|-\left|J_{A F 2}^{*}\right|<2 H^{c} .
\end{aligned}
$$

Therefore, large coercivities will increase the range of operation of the ratchet scheme in terms of the coupling strengths that can be used. These equations show that for coupled magnetic layers designed as set out here, an oscillating perpendicular magnetic field can drive a soliton unidirectionally up the stack. The ferromagnetic coupling provides the necessary broken spatial symmetry for ratchet behaviour. However, this does not lead directly to the ratchet operation, which also depends on how the switching of one layer changes the energy of neighboring layers and the interaction of this with the oscillating applied magnetic field.

We demonstrate this ratchet scheme experimentally using $\mathrm{Pt} / \mathrm{Co}$ magnetic layers which provide large perpendicular anisotropy and sharp coercive switching, approximating the Ising-macrospin behavior. ${ }^{13}$ The Co layers are coupled using RKKY interactions through $\mathrm{Pt} / \mathrm{Ru} / \mathrm{Pt}$ spacers. In order to calibrate the three different coupling strengths, we grew a series of bilayer and trilayer samples (see also supplementary information). ${ }^{17}$ Figure 2(a) shows a schematic of the bilayers used to determine the antiferromagnetic coupling strength. We are able to control the AF coupling strength by varying the $\mathrm{Pt}$ thickness either side of the Ru layer, ${ }^{21}$ as shown in Figure 2(a). For measurement of F coupling, we use a trilayer stack as shown in Figure 2(b), with this more complicated structure necessary to prevent the two ferromagnetically coupled layers switching together. ${ }^{20}$ By controlling the Ru thickness, we can find the peak in the ferromagnetic coupling between the first and second antiferromagnetic peaks.

We now experimentally demonstrate soliton propagation using MOKE magnetometry. The multilayer stack is shown schematically in Figure 3(a). We use $\mathrm{Ta}(4) / \mathrm{Pt}(10)$ buffer 

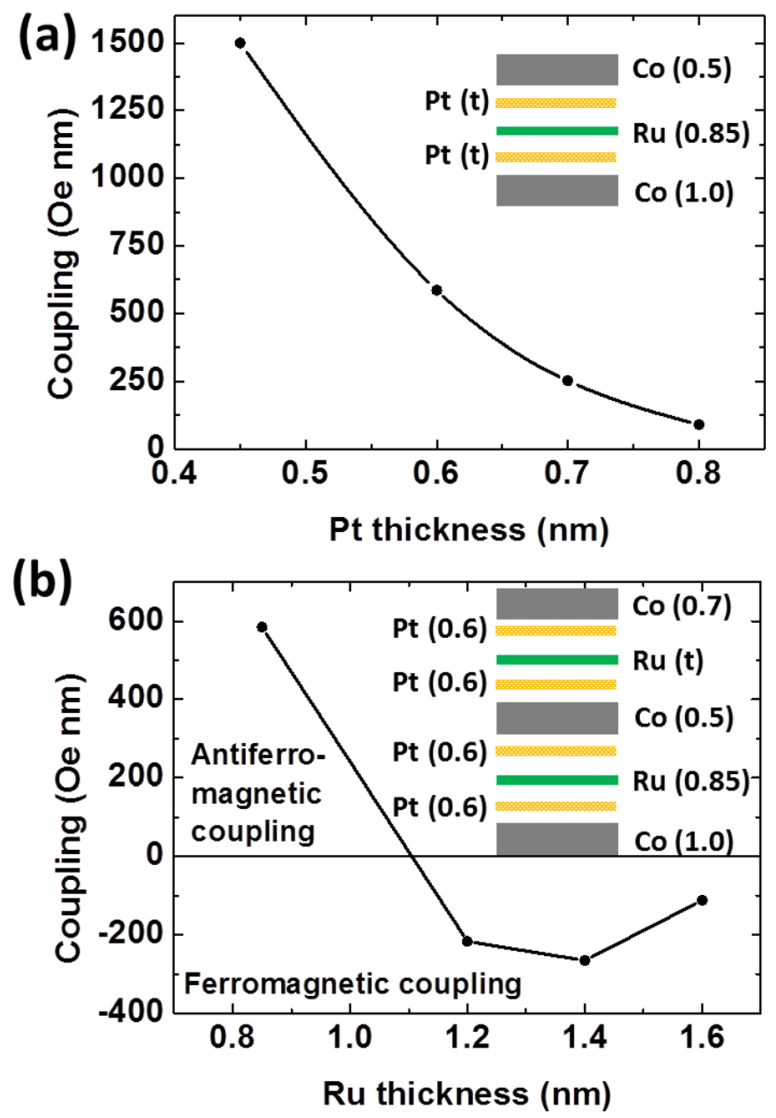

FIG. 2. (a) Antiferromagnetic coupling strength as function of Pt interlayer thickness. The inset shows a schematic of the stack used to measure the coupling. (b) Extracted coupling strength as a function of Ru thickness, using the stack shown in the inset. All thicknesses are in nm.

layers (all thicknesses in $\mathrm{nm}$ ) before adding eight Co $0.6 \mathrm{~nm}$ thick layers with two repeats of the AF1-AF2-F RKKY coupling scheme. For the stronger AF1 coupling we use $\mathrm{Pt}(0.68) / \mathrm{Ru}(0.85) / \mathrm{Pt}(0.68)$ layers; for the AF2 coupling we use $\mathrm{Pt}(0.80) / \mathrm{Ru}(0.85) / \mathrm{Pt}(0.80)$ layers and for the $\mathrm{F}$ coupling we use $\mathrm{Pt}(0.6) / \mathrm{Ru}(1.4) / \mathrm{Pt}(0.6)$. Due to the skin depth of the laser, the relative magnitude of the Kerr signal decreases for layers nearer the bottom of the stack. Because we have identical magnetic layers, we can use this as a guide to which layer is switching. One important consideration is to be able to inject a soliton into the system. ${ }^{8,16}$ For simplicity, we use a scheme where we add another AF1 coupled layer at the bottom of the stack as shown in Figure 3(a) in order to create a soliton at remanence when coming from saturation. Coming from saturation (A) layer 2 will be the first to switch (B) as shown in Figure 3(b), which gives the major loop of the stack (black). This in turn means that layer 3 is now stabilized by the AF1 coupling to layer 2. The next layer to switch is the only one with two antiferromagnetic couplings to neighboring layers, layer $6(\mathrm{C})$. After this switch, at remanence, we have created a soliton, indicated by the blue star in Figure 3(a), consisting of two layers, 3 and 4, parallel aligned across an AF2 coupling. Then in the major loop we see that we have combined switching of layers 4 and 5 (D), then 7 and 8 (E). Finally, layers $1(\mathrm{~F})$ and $3(\mathrm{G})$ switch, which have to overcome high AF coupling and so switch at the highest fields.

In order to propagate a soliton (shown in red in Figure 3(b)) we start at remanence (C), then increase the field until
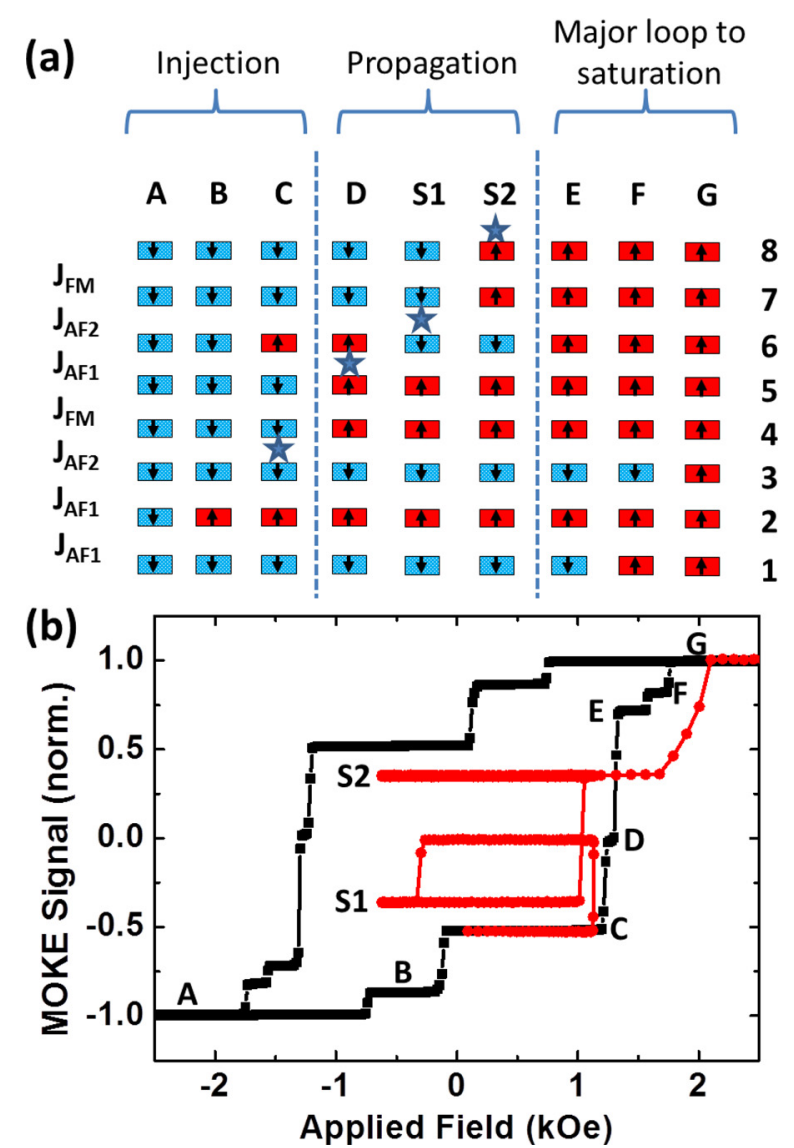

FIG. 3. (a) Schematic showing the magnetization directions of the layers during the major loop and soliton propagation, with the interlayer coupling labelled to the left and the Co layer number to the right. The different layer configurations are labelled above. The position of the soliton is labelled by the blue star. (b) MOKE data showing the major loop of the sample (black) and soliton propagation (red).

layers 4 and 5 switch (D) together and then reduce the field magnitude. This leaves the sample in state D with a soliton consisting of layers 5 and 6 . On reducing the field we reach a point where the total moment reduces again as layer 6 reverses (S1). We can see that the state reached is one that does not occur in the major loop, which is that of a soliton between layers 6 and 7. We then increase the field to the same field that switched layers 4 and 5 and now layers 7 and 8 switch, which expels the soliton from the stack. Cycling the field back and forth leads to no further changes since by expelling the soliton we have reached the ground state of the system. The fact that the two F coupled layers switch together means that we have a two step ratchet which is functionally equivalent to one which uses two different magnetic layer thicknesses alternating with two different AF couplings, as demonstrated previously. ${ }^{1}$ We can use minor loops to extract the coercivities and coupling strengths. ${ }^{1,16}$ which are given in Table I (see also supplementary material). ${ }^{17} \mathrm{We}$ assume here that the $\mathrm{F}$ coupled layers behave as a single layer with twice the thickness. We did, however, design the system to have an $J_{F M}$ of magnitude 250 Oe nm (the maximum in Figure 2(b)) which as can be seen from Table I is much closer in magnitude to $J_{A F 2}$ than $J_{A F 1}$. This should have led to the possibility of a soliton across the $\mathrm{F}$ coupling, and so a three-step ratchet behavior (see also Eqs. (4) and (6)). There are several possibilities to explain this behavior. First, the F coupling is stronger than 
TABLE I. Extracted coercivities and interlayer couplings of the soliton stack. The couplings are listed in the same order as in Figure 3(a). An asterisk (*) indicates that no value for the coupling could be obtained.

\begin{tabular}{lccc}
\hline \hline Co layer & $H^{c}(\mathrm{Oe})$ & Coupling layer & Coupling $(\mathrm{Oe} \mathrm{nm})$ \\
\hline $7+8$ & 923 & $J_{F M}$ & $*$ \\
6 & 876 & $J_{A F 2}$ & 209 \\
$4+5$ & 996 & $J_{A F 1}$ & 374 \\
3 & 516 & $J_{F M}$ & $*$ \\
2 & 731 & $J_{A F 2}$ & 236 \\
1 & 799 & $J_{A F 1}$ & 505 \\
& & $J_{A F 1}$ & 415 \\
\hline \hline
\end{tabular}

expected, possibly due to an increase in roughness, and therefore pinholes, as the stack height increases. ${ }^{22}$ This would cause domains in both ferromagnetically coupled layers to nucleate simultaneously and a coupled domain wall to move across the film, which we have seen in MOKE microscopy (see supplementary material). ${ }^{17}$ Even without pinholes, collective switching of two F coupled layers is likely, since both Zeeman energy and the coupling will favour this, unlike the AF coupled case. The nucleation of domains is dominated by the most ferromagnetic defects for both the F coupled layers, which is not true in all cases for AF coupled layers, for example, layer 6 during soliton propagation, where nucleation occurs at the most antiferromagnetic defects. ${ }^{22}$ The data in Table I also show that the ratchet scheme was able to operate even with a fairly wide dispersion of both coercivity and coupling in this particular stack indicating the robustness of soliton propagation to such random variation.

In conclusion, we have experimentally demonstrated the vertical propagation of a soliton in a repeated AF-AF-F coupled superlattice, having derived the conditions for correct soliton propagation in such a structure assuming an Isingmacrospin behavior. The combined switching of the two ferromagnetic layers seen experimentally means that this design is functionally equivalent to the previously demonstrated vertical ratchet schemes. This work shows the possibility of creating ratchet devices using mixed ferromagnetic and antiferromagnetic coupling with unvaried magnetic layers which allow out-of-plane propagation of data.

This research was funded by the European Community under the Seventh Framework Program ERC Contract No.
247368: 3SPIN. R.L. acknowledges support from the Netherlands Organization for Scientific Research (VENI 68047428). A.F.-P. acknowledges an EPSRC Early Career fellowship and support from the Winton Programme for the Physics of Sustainability.

${ }^{1}$ R. Lavrijsen, J. H. Lee, D. Petit, A. Fernández-Pacheco, R. Mansell, and R. P. Cowburn, Nature 493, 647 (2013).

${ }^{2}$ S. S. P. Parkin, M. Hayashi, and L. Thomas, Science 320, 190 (2008).

${ }^{3}$ A. Fernández-Pacheco, L. Serrano-Ramón, J. M. Michalik, M. R. Ibarra, J. M. D. Teresa, L. O'Brien, D. Petit, J. Lee, and R. P. Cowburn, Sci. Rep. 3, 1492 (2013).

${ }^{4}$ D. Chiba, G. Yamada, T. Koyama, K. Ueda, H. Tanigawa, S. Fukami, T. Suzuki, N. Ohshima, N. Ishiwata, Y. Nakatani, and T. Ono, Appl. Phys. Express 3, 073004 (2010).

${ }^{5}$ D. A. Allwood, G. Xiong, C. C. Faulkner, D. Atkinson, D. Petit, and R. P. Cowburn, Science 309, 1688 (2005).

${ }^{6}$ R. P. Cowburn and M. E. Welland, Science 287, 1466 (2000).

${ }^{7}$ K.-J. Kim, J.-C. Lee, S.-J. Yun, G.-H. Gim, K.-S. Lee, S.-B. Choe, and K.H. Shin, Appl. Phys. Express 3, 083001 (2010).

${ }^{8}$ A. Fernández-Pacheco, D. Petit, R. Mansell, R. Lavrijsen, J. H. Lee, and R. P. Cowburn, Phys. Rev. B 86, 104422 (2012).

${ }^{9}$ R. Skomski, Z. Li, R. Zhang, R. D. Kirby, A. Enders, D. Schmidt, T. Hofmann, E. Schubert, and D. J. Sellmyer, J. Appl. Phys. 111, 07E116 (2012).

${ }^{10}$ R. P. Cowburn, Patent WO2012076871A1 (14 June 2012).

${ }^{11}$ D. Petit, R. Mansell, A. Fernández-Pacheco, J. Lee, and R. Cowburn, "Three-dimensional spintronics," in VLSI: Circuits for Emerging Applications, Devices, Circuits, and Systems, edited by T. Wojcicki (CRC Press, 2014), Chap. 12.

${ }^{12}$ E. Y. Vedmedenko and D. Altwein, Phys. Rev. Lett. 112, 017206 (2014).

${ }^{13}$ J. Ferré, T. Devolder, H. Bernas, J. P. Jamet, V. Repain, M. Bauer, N. Vernier, and C. Chappert, J. Phys. D: Appl. Phys. 36, 3103 (2003).

${ }^{14}$ J. H. Franken, H. J. M. Swagten, and B. Koopmans, Nat. Nanotechnol. 7, 499 (2012).

${ }^{15}$ J. Kiermaier, S. Breitkreutz, I. Eichwald, M. Engelstdter, X. Ju, G. Csaba, D. Schmitt-Landsiedel, and M. Becherer, J. Appl. Phys. 113, $17 \mathrm{~B} 902$ (2013).

${ }^{16}$ R. Lavrijsen, D. C. M. C. Petit, A. Fernández-Pacheco, J. Lee, R. Mansell, and R. P. Cowburn, Nanotechnology 25, 105201 (2014).

${ }^{17}$ See supplementary material at http://dx.doi.org/10.1063/1.4914014 for details on the three step AF ratchet, determining the F and AF coupling strengths, minor loops and the collective switching of F coupled layers.

${ }^{18}$ P. Bruno, G. Bayreuther, P. Beauvillain, C. Chappert, G. Lugert, D. Renard, J. P. Renard, and J. Seiden, J. Appl. Phys. 68, 5759 (1990).

${ }^{19}$ A. Fernández-Pacheco, F. C. Ummelen, R. Mansell, D. Petit, J. H. Lee, H. J. M. Swagten, and R. P. Cowburn, Appl. Phys. Lett. 105, 092408 (2014).

${ }^{20}$ P. J. Metaxas, R. L. Stamps, J.-P. Jamet, J. Ferré, V. Baltz, B. Rodmacq, and P. Politi, Phys. Rev. Lett. 104, 237206 (2010).

${ }^{21}$ R. Lavrijsen, A. Fernández-Pacheco, D. Petit, R. Mansell, J. H. Lee, and R. P. Cowburn, Appl. Phys. Lett. 100, 052411 (2012).

${ }^{22}$ J.-H. Lee, R. Mansell, D. Petit, A. Fernández-Pacheco, R. Lavrijsen, and R. Cowburn, SPIN 3, 1340013 (2013). 